\title{
Ernährung im Alltag
}

\section{Problematisierungen dominieren die Diskussionen über Ernährung in Medien und Politik. Thematisiert werden die Folgen von Übergewicht bei Kindern, die Ab- nahme von Ernährungskompetenzen oder die Zunahme ernährungsmitbedingter Krankheiten und ein Verlust von Ernährungskultur, insbesondere die Folge- kosten für das Gesundheitswesen. Skandalisierungen und Pauschalurteile werden jedoch den KonsumentInnen nicht gerecht. Dies zeigt ein differenzierter Blick auf den Ernährungsalltag.}

lichen Mahlzeiten strukturieren den Tag und liefern eine Orientierung für die übrige Alltagsgestaltung. Dies schlägt sich im Ernährungsverhalten nieder: häufige gemeinsame häusliche Mahlzeiten und tägliches Kochen, wenige Außer HausMahlzeiten und geringe Nutzung von Convenience-Angeboten. Die freudlosen GewohnheitsköchInnen repräsentieren das älteste Segment der Typologie. Die Mehrheit befindet sich im Ruhestand oder war nie berufstätig. Über die Hälfte ist verheiratet, ein Drittel verwitwet.

Die fitnessorientierten Ambitionierten zeichnen sich dadurch aus, dass sie mit einer hochwertigen und disziplinierten Ernährung Leistungsfähigkeit und Fitness des Körpers erhalten wollen. Berufli-
$\mathrm{U}$ Von Doris Hayn $m$ den Ernährungsalltag in den Blick zu nehmen, hat das Institut für sozial-ökologische Forschung (ISOE) im Frühjahr 2004 eine repräsentative Befragung von 2.039 Personen ab 18 Jahren durchgeführt. Mit Hilfe des sozial-ökologischen Lebensstilansatzes wurden sowohl Ernährungsorientierungen als auch -verhalten detailliert untersucht. Dabei wurden Orientierungen bezogen auf Ernährung allgemein, auf mit Ernährung und Versorgung verbundene Aktivitäten (Einkaufen, Kochen, Mahlzeiten) und auf ausgewählte Produkte und Produktqualitäten erfragt. Darüber hinaus wurden allgemeine Lebensstilorientierungen, soziodemografische und sozialstrukturelle Merkmale sowie differenzierte Angaben zur beruflichen Tätigkeit erfragt. Auf Basis der erhobenen Daten wurde eine Typologie von sieben Ernährungsstilen entwickelt (1).

\section{- Die Ernährungsstile der Deutschen}

Zentrale Kennzeichen der desinteressierten FastFooder sind ein ausgeprägtes Desinteresse an Ernährungs- und Gesundheitsfragen sowie ein hohes Maß an Flexibilisierung und Enthäuslichung. Festen Essensrhythmen wird wenig Bedeutung beigemessen, das Interesse am Kochen ist gering. Essen außer Haus hat dagegen einen hohen Stellenwert. Diese geringe Bedeutung von Ernährung spiegelt sich deutlich im Verhalten wider: Zuhause gekocht wird selten, die Ernährung spielt sich größtenteils in Fast Food-Restaurants und Mensen ab. Frisches Gemüse und Obst wird selten, Fleisch sowie Produkte mit gesundheitsfördernden Zusätzen werden überdurchschnittlich oft verzehrt. Dieser Ernährungsstil ist vor allem bei jüngeren Singles und Paaren verbreitet, Männer sind überdurchschnittlich vertreten.

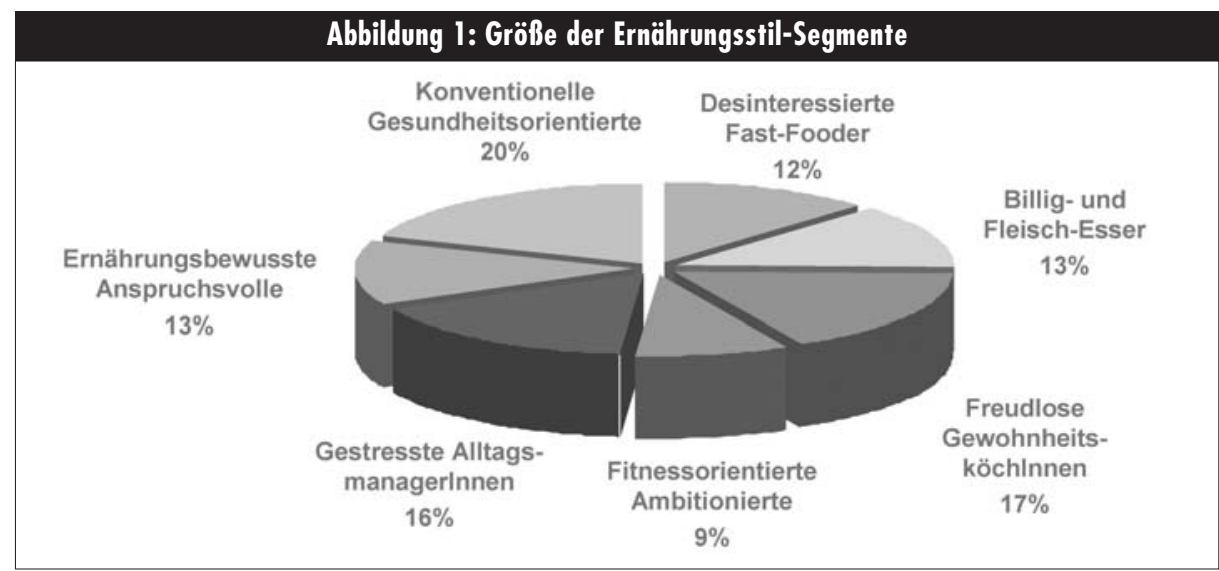

Dominierendes Motiv der Billig- und Fleisch-Esser ist, dass Ernährung preiswert und unkompliziert sein muss. Gesundheit spielt eine untergeordnete Rolle, gegessen wird nach Lust und Laune. Das Bedürfnis nach Vereinfachung zeigt sich in der Auflösung von Ernährungsroutinen, dem Bedeutungsverlust von gemeinsamen Mahlzeiten und der Bevorzugung von Convenience-Lösungen. Fleisch gilt als ideales Nahrungsmittel, da es einfach und zugleich kreativ zubereitet werden kann. Diese Orientierungen schlagen sich im Verhalten nieder: gekocht wird nur gelegentlich und dann häufig mit Fertiggerichten. Der Speiseplan zeichnet sich durch einen hohen Anteil an Fleisch und Wurst aus, Obst und Gemüse werden selten verzehrt. Der Schwerpunkt der Billig- und Fleischesser liegt bei der mittleren Altersgruppe. Der Ernährungsstil ist jedoch auch bei den 46-60-Jährigen und den unter 25-Jährigen verbreitet und findet sich bei Singles, ebenso wie bei Paaren und Familien.

Herausragende Kennzeichen der freudlosen GewohnheitsköchInnen sind ein gering ausgeprägtes Interesse an Ernährung und fest verankerte Ernährungsgewohnheiten. Essen hat den Charakter einer Pflichterfüllung, ist kaum mit Freude und Genuss verbunden. Die regelmäßigen häus-
Quelle: ISOE

che und private Anforderungen sollen mit einer gesunden Ernährung ausgeglichen werden. Dabei spielen hochwertige und gesundheitsfördernde Produkte wie Bio-Lebensmittel, aber auch Functional Food eine wichtige Rolle. Die Bereitschaft zu Kompromissen kennzeichnet insgesamt das Ernährungsverhalten. Kreatives, entspannendes Kochen am Wochenende stellt einen Ausgleich dar zur häufigen Nutzung von Außer Haus-Angeboten im Berufsalltag. Flexible Essenszeiten werden akzeptiert, solange täglich eine gemeinsame Mahlzeit im Haushalt möglich ist. Fitnessorientierte Ambitionierte finden sich überwiegend bei Paaren und Familien. Der Altersschwerpunkt liegt bei den 4050-Jährigen. In vielen Haushalten sind beide Partner berufstätig. Viele arbeiten als FreiberuflerInnen und Selbstständige, entsprechend hoch ist das durchschnittliche Einkommen.

Kennzeichnend für die gestressten AlltagsmanagerInnen ist ein aufreibender Spagat zwischen hohen Ansprüchen an Ernährung und Kräfte zehrenden Anforderungen des familiären und beruflichen Alltags. Das ausgeprägte Interesse an Ernährungsfragen ist begründet im Bestreben, den Kindern bzw. der Familie eine optimale Ernährung zu bieten: so 
soll regelmäßig, abwechslungsreich und mit frischen Zutaten gekocht werden. Da es an Zeit sowie an Unterstïtzung durch andere Haushaltsmitglieder mangelt, lässt sich dieser Anspruch jedoch schwer aufrechterhalten: Einkauf und Kochen werden zur lästigen Pflicht. Entlastungsmöglichkeiten wie Convenience-Produkte laufen häufig den Ansprüchen zuwider und übersteigen oft die finanziellen Möglichkeiten. Dieser Ernährungsstil weist den deutlichsten geschlechtsspezifischen Charakter auf: Etwa drei Viertel sind Frauen, die meist allein die Verantwortung für die Ernährung der gesamten Familie tragen.

Grundlegend für die ernährungsbewussten Anspruchsvollen ist ein ausgeprägtes Interesse an Ernährungsfragen sowie ein ganzheitlicher Blick auf Ernährung und Gesundheit. Geachtet wird auf Qualität, Frische, Regionalität und Naturbelassenheit der Lebensmittel. Bio-Lebensmittel gelten als Wohltat für Körper und Seele, synthetische Zusatzstoffe werden strikt abgelehnt. Ein Augenmerk liegt auf Genuss und kommunikativer Esskultur. Dass diese Ansprüche realisiert werden, zeigt sich unter anderem am hohen Obst- und Gemüseverzehr und am niedrigen Fleischkonsum. Ernährung wird Zeit und Raum im Alltag eingeräumt, sie ist ein anspruchsvoller, eigenständiger Gestaltungsbereich. Dieser Ernährungsstil ist weder an eine bestimmte Lebensphase noch an eine bestimmte Altersgruppe gebunden.

Die konventionellen Gesundheitsorientierten haben eine hohe Wertschätzung für gutes Essen und starkes Interesse an Ernährung. Essen in kommunikativer Atmosphäre und der Einkauf sind positiv besetzt. Gekocht wird gerne und reichlich. Die konventionellen Gesundheitsorientierten sind eine Gruppe von ,neuen Älteren“, deren Genuss und Freude am Essen vom Kampf mit Gewichtsund Gesundheitsproblemen gedämpft wird. Der eigene Anspruch auf Süßes zu verzichten und weniger Fleisch zu essen, kann im Alltag nicht konsequent umgesetzt werden. Dieser Ernährungsstil ist bei Paaren und Alleinlebenden in der Nachfamilienphase verbreitet.

Die Typologie zeigt deutlich die Vielfalt der Ernährungsstile und liefert damit ein differenziertes Bild des Ernährungsalltags der Deutschen. Sie belegt, dass aktuell diskutierte Trends wie Enthäuslichung, Entrhythmisierung, Convenienceund Fast-Orientierung keine „Breitenphänomene“ sind. Einzelne Trends spiegeln sich im Alltag mancher Ernährungsstile wider, sind eingebettet in spezifische Alltagsarrangements, die jeweils ihre eigene Rationalität besitzen. Die Typologie

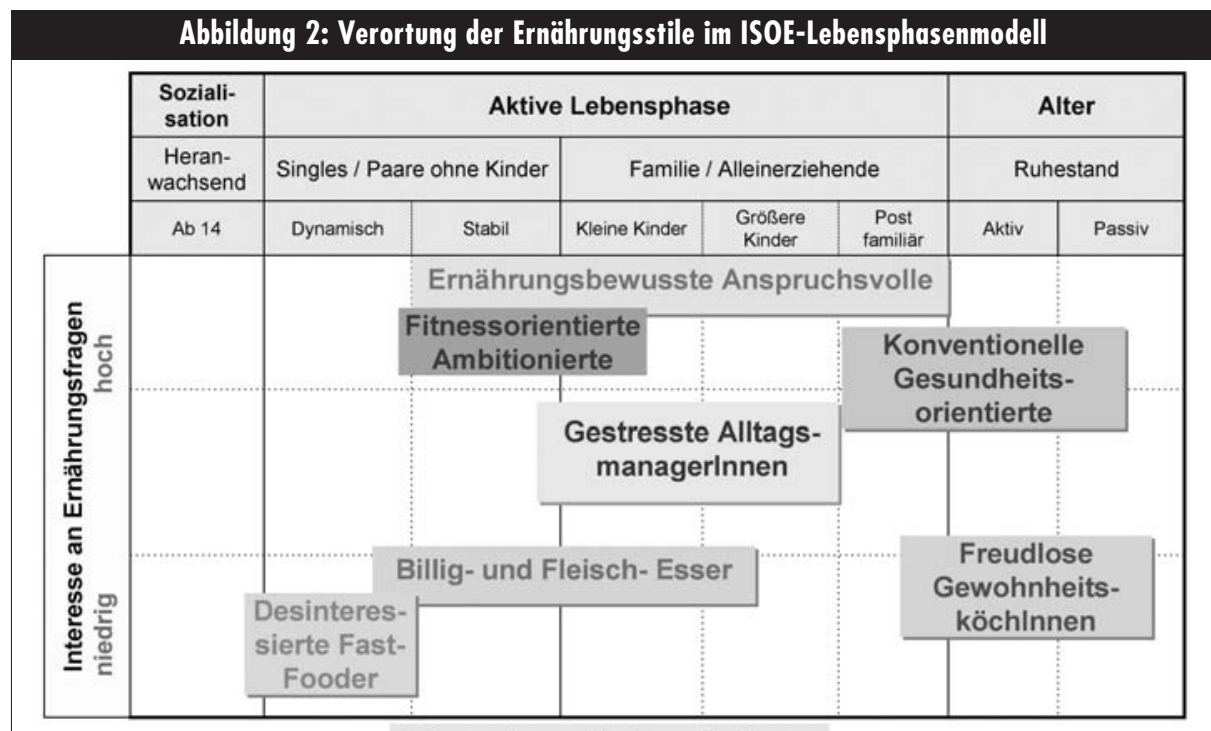

Lebensphasen / Lebenssituationen

zeigt, wie Ernährungs- und Gesundheitsorientierungen mit Anforderungen und Ansprüchen anderer Lebensbereiche, vor allem Beruf und Freizeit, zusammengebracht, in konkretes Ernährungshandeln umgesetzt und so zu einem ,stimmigen Ganzen“ integriert werden: dem jeweiligen Ernährungsstil.

\section{- Veränderungen im Lauf des Lebens}

Dass sich die Ernährungsgewohnheiten im Laufe des Lebens verändern können, wird sichtbar, wenn die Ernährungsstile in ein Lebensphasenmodell eingeordnet werden. Insbesondere bei den desinteressierten Fast-Foodern handelt es sich um einen Ernährungsstil, der an eine bestimmte Lebensphase, die Vorfamilienphase, gebunden ist (siehe Abbildung 2). Dies zeigt die Bedeutung von biografischen Übergängen: mit der Geburt von Kindern, aber auch dem Zusammenziehen mit einem Partner ändert sich die Versorgungssituation und damit der Ernährungsalltag. Dass sich die desinteressierten Fast-Fooder fast ausschließlich bei den 18-25-Jährigen finden, verweist ferner auf die Relevanz der Ernährung in der vorausgehenden Lebensphase. Jugendliche scheinen ihren ersten eigenen Haushalt mit Ernährungsgewohnheiten und -erfahrungen zu gründen, die sie - wenn auch nicht dauerhaft - zu „Außer-Haus-Essern“ macht. Dies wirft die Frage nach einer adäquaten Ernährungsversorgung und Kompetenzvermittlung durch die Schule auf.

Abbildung 2 zeigt darüber hinaus, dass die Ernährungsstile mit Blick auf das Interesse an Ernährungsfragen (horizontale Achse) in zwei Gruppen unterteilt werden können. Bei den desinteressierten Fast-Foodern, den Billig- und
Quelle: ISOE

Fleisch-Essern sowie den freudlosen GewohnheitsköchInnen ist das Interesse an Ernährungsfragen gering ausgeprägt. Demgegenüber haben die anderen vier Ernährungsstile ein stark ausgeprägtes Interesse. Dies gibt Hinweise für die Entwicklung von Handlungsstrategien: Bei der zweiten Gruppe kann an das vorhandene Interesse angeknüpft werden, bei der ersten Gruppe müssen dagegen Strategien erarbeitet werden, die entweder Allianzen mit anderen Motiven (z.B. Convenience) herstellen oder auf Angebote zielen, mit denen existierende Hemmnisse abgebaut oder entschärft werden können.

Die Typologie der Ernährungsstile liefert konkrete Hinweise auf Potenziale aber auch auf Hemmnisse in Richtung nachhaltigerer und gesünderer Ernährung. Sie wird im Projekt Ernährungswende als ein wesentlicher Ausgangspunkt für die Entwicklung zielgruppenbezogener Kommunikations- und Beratungsangebote sowie alltagsadäquater Produkte und Dienstleistungen genutzt.

\section{Anmerkung}

(1) Eine ausführlichere Beschreibung der Ernährungsstile wird als Diskussionspapier unter www.ernaehrungswende.de veröffentlicht.

\section{Die Autorin}

Dr. Doris Hayn ist wissenschaftliche Mitarbeiterin im Institut für sozial-ökologische Forschung (ISOE). Kontakt: ISOE, Hamburger Allee 45, 60486 Frankfurt/ M. Tel. 069-707691931, E-Mail: hayn@isoe.de 
(c) 20I0 Authors; licensee IÖW and oekom verlag. This is an article distributed under the terms of the Creative Commons Attribution Non-Commercial No Derivates License (http://creativecommons.org/licenses/by-nc-nd/3.o/), which permits unrestricted use, distribution, and reproduction in any medium, provided the original work is properly cited. 